\title{
Câncer de mama e meio ambiente: uma revisão integrativa
}

\section{Breast cancer and environment: an integrative review}

\section{Igor Marcelo Castro e Silva ${ }^{1}$, Eloisa da Graça do Rosário Gonçalves ${ }^{2}$}

${ }^{1}$ Mestrando em Saúde e Ambiente pela Universidade Federal do Maranhão e Docente do Departamento de Patologia da Universidade Federal do Maranhão.

${ }^{2}$ Docente orientadora do Curso de Mestrado em Saúde e Ambiente da Universidade Federal do Maranhão.

\section{Resumo}

Introdução: O Câncer de mama acomete as mulheres tanto no mundo ocidental, quanto oriental. Remete, principalmente, aos grupos etários economicamente ativos e as em idade fértil, configurando fato preocupante à saúde pública. Objetivo: Realizar uma revisão integrativa do câncer de mama com enfoque em sua epidemiologia, estadiamento, manifestações clínicas, tratamento e influência do ambiente na referida neoplasia. Métodos: Revisão bibliográfica integrativa, incluindo trabalhos originais ou de revisão. O câncer de mama é o tipo de câncer que acomete com maior frequência as mulheres. No Brasil, para o biênio 2016-2017, esperam-se 57.916 casos novos de câncer da mama, com um risco estimado de 52 casos a cada 100 mil mulheres. O câncer de mama denota considerável ameaça, carregando consigo o preconceito, a discriminação e solidão, efeitos estes geradores de preocupação entre os profissionais de saúde. Associado a todos os caracteres peculiares dessa patologia, alia-se os diversos tratamentos tanto cirúrgicos quanto quimioterápicos. Suas indicações refletem em vários âmbitos na qualidade de vida da paciente, induzindo, geralmente, a desconfortos físicos, emocionais, espirituais, econômicos e sociais. Conclusão: O Câncer de mama detém altas taxas de mortalidade, mesmo com o desenrolar das terapias, justificando-se pela heterogeneidade da doença. Urge necessidade de maiores estudos e investimentos acerca de tal problemática, por se constituir, na atualidade, de um imbróglio à saúde pública.

Palavras-chave: Câncer de Mama; Neoplasias da mama; Saúde da Mulher.

Autor correspondente:

Prof. Igor Marcelo Castro e Silva

Endereço: Praça Madre de Deus 01, Bairro Madre Deus

Cep: 65025-560 - São Luís, MA-Brasil

Telefone: $+55313319-4269$

+553199380-2020

Recebido em: 30/01/2017

Revisado em: 06/04/2017

Aceito em: 16/04/2017

E-mail: igormarcelo23@hotmail.com

Publicado em: 28/04/2017 


\section{Abstract}

Introduction: Breast cancer affects women in the world. It refers mainly to the economically active age groups and those of childbearing age. Objective: To carry out an integrative review regarding breast cancer and the environment. Methods: Integrative bibliographic review, included were original or revision work. Breast cancer is the type of cancer that affects women more often. In Brazil, for the biennium 2016-2017, 57,916 new cases of breast cancer are expected, with an estimated risk of 52 cases per 100,000 women. Breast cancer denotes a considerable threat, carrying with it prejudice, discrimination and loneliness, which are the causes of concern among health professionals. Associated with all the peculiar characteristics of this pathology, the various treatments, both surgical and chemotherapeutic, are combined. Its indications reflect in various scopes in the quality of life of the patient, generally inducing physical, emotional, spiritual, economic and social discomforts. Conclusion: Breast cancer has high mortality rates, even with the development of therapies, justifying the heterogeneity of the disease. There is a need for further studies and investments on this issue, because it is currently a public health problem.

Key words: Breast Cancer; Breast Neoplasms; Women's Health.

\section{Introdução}

No decorrer da História, verificou-se que, no século XIX e XX, ocorreram diversas e importantes inovações tecnológicas e científicas que modificaram a civilização ocidental, gerando mudanças ímpares nas condições de vida das populações ${ }^{1}$.

Grande parte da transição a uma melhora qualidade de vida deveu-se, principalmente, aos desenvolvimentos das ciências da saúde. A Revolução Pausteriana, demonstrando a existência de microorganismos causadores de doença e a possibilidade de vacinas e soros, o nascimento da epidemiologia, baseada no estudo do cólera em Londres, realizado pelo médico inglês John Snow ${ }^{2}$, a elucidação da estrutura do DNA ${ }^{3}$, entre outras descobertas e invenções, contribuíram ao aumento da expectativa de vida e, com esta, das doenças crônicas.

Acerca das doenças crônicas, na atualidade, o câncer encontra-se com uma elevada incidência de mortalidade em nível mundial e Brasil. O câncer, em si, é uma doença de múltiplos fatores, e evidencia um interlace expressivo entre genes, estilo de vida e meio ambiente, que podem atuar, geralmente, de forma combinada. Inúmeros eventos endógenos e exógenos podem levar direta ou indiretamente a mutações ou elevar a probabilidade dessas ações e o desenvolvimento do câncer. Contidos nessa lista estão as radiações ionizantes, os produtos químicos, algumas bactérias, vírus, hábitos alimentares ${ }^{4,13}$.

Epidemiologicamente, o câncer configura-se como a segunda causa de morte por doença na maioria dos países desenvolvidos e os em desenvolvimento, os mais frequentes são os de pulmão, cólon e mama feminina ${ }^{5}$.

O Câncer de mama acomete com maior regularidade as mulheres tanto no mundo ocidental, quanto oriental. Remete, principalmente, aos grupos etários economicamente ativos, as mulheres em idade fértil, tornou-se um fato preocupante à saúde pública. Paralelamente, observa-se que o diagnóstico precoce em ascensão, resultante da associação das campanhas de prevenção, tecnologia de ponta utilizadas no contexto câncer, das terapêuticas com maior especificidade, permitiu o aumento da sobrevida global e livre de doença dessas mulheres ${ }^{6}$.

Determinada neoplasia, estando em tratamento ou não, em sua essência, denota considerável ameaça, carregando consigo o preconceito, a discriminação e a solidão ${ }^{7}$. Assim, o aumento dos anos de vida das mulheres com câncer de mama não garante permanência de boa qualidade de vida. Com todos os avanços inerentes ao tratamento antineoplásico, este, contudo, provoca toxicidade, excitando a exaustão aguda e crônica em função da citotoxicidade dos seus agentes. Aliado ao contexto, pode originar diminuição nas capacidades fisiológicas, cognitivas e sociais, contribuindo a uma piora da Qualidade de Vida e sobrevida da paciente.

O presente estudo, assim, teve por objetivo principal realizar uma revisão integrativa do câncer de mama e meio ambiente, com enfoque em sua epidemiologia, stadiamento, manifestações clínicas, tratamento e o envolvimento ambiente, doença, saúde. 


\section{Câncer de mama: conceitos}

O câncer de mama é uma das doenças mais receadas entre as mulheres, principalmente, no grupo etário após os 35 anos de idade, onde sua incidência cresce de maneira progressiva e devastadora ${ }^{8}$.

A magnitude do câncer de mama é de extrema grandiosidade, traduzindo-se em uma patologia vastamente estudada pelo meio científico. Segundo o banco de dados Pubmed, no primeiro semestre de 2016, foram publicados, entre artigos originais e de revisão, 17683 artigos científicos em todo o mundo.

\section{Epidemiologia}

Segundo INCA ${ }^{9}$, o câncer é, sem dúvidas, um problema de saúde pública, principalmente, entre os países em desenvolvimento, onde é esperado que o impacto do câncer na população corresponda a $80 \%$ dos mais de 20 milhões de casos novos estimados para 2025.

A estimativa mundial, realizada em 2012, pelo projeto Globocan/Iarc, apontou que os tipos de câncer mais incidentes no mundo foram pulmão (1,8 milhão), mama (1,7 milhão), intestino (1,4 milhão) e próstata (1,1 milhão). Nos homens, os mais frequentes foram pulmão $(16,7 \%)$, próstata $(15,0 \%)$, intestino $(10,0 \%)$, estômago $(8,5 \%)$ e fígado $(7,5 \%)$. Em mulheres, as maiores frequências encontradas foram mama $(25,2 \%)$, intestino $(9,2 \%)$, pulmão $(8,7 \%)$, colo do útero $(7,9 \%)$ e estômago $(4,8 \%)^{9}$.

O número de mortes, em nível de Brasil, correspondeu a 14.388, 181 homens e 14.206 mulheres, para o ano de 2013. Em se tratando do Câncer de Mama, para o biênio 2016-2017, há uma estimativa de 57.960 casos novos, 11.960 para a região nordeste ${ }^{9}$.

\section{Estadiamento}

O estadiamento do Câncer de Mama possui papel essencial em todas as fases do tratamento, assim como, o real prognóstico evolutivo da doença. O processo de estadiamento engloba conceitos que integram a visão objetiva e coesa do paciente, dos dados clínicos e patológicos obtidos.

O câncer de mama é estadiado baseado no Sistema TNM, recomendado pelo American Joint Commitee on Cancer and International Union for Cancer Control ( AJCC- UICC) ${ }^{10}$.

O sistema TNM classifica os tumores segundo os atributos histológicos que influenciam diretamente no prognóstico: tamanho do tumor $(\mathrm{T})$, presença e extensão do acometimento linfonodal locorregional $(\mathrm{N})$ e presença ou ausência de metástases $(\mathrm{M})$.

Após a determinação do tamanho do tumor, da invasão linfática e metastização presente ou ausente, estabelece-se o estadio atual da doença, como explicitado nas figuras $01 \mathrm{e} 02$.

\begin{tabular}{|c|c|}
\hline \multirow{2}{*}{\multicolumn{2}{|c|}{ T - Tumor }} \\
\hline & \\
\hline TX & O tumor primário não pode ser avaliado \\
\hline TO & Sem evidência de tumor primário \\
\hline Tis & $\begin{array}{l}\text { Carcinoma in situ: carcinoma intraductal ou carcinoma lobular in situ ou doença de Paget } \\
\text { da papila sem tumor }\end{array}$ \\
\hline \multirow[t]{4}{*}{ T1 } & Tumor com $2 \mathrm{~cm}$ ou menos em sua maior dimensão \\
\hline & T1a - tumor com $0,5 \mathrm{~cm}$ ou menos em sua maior dimensão \\
\hline & T1b - tumor com mais de $0,5 \mathrm{~cm}$ e até $1 \mathrm{~cm}$ em sua maior dimensão \\
\hline & T1c - tumor com mais de $1 \mathrm{~cm}$ e até $2 \mathrm{~cm}$ em sua maior dimensão \\
\hline T2 & Tumor com mais de $2 \mathrm{~cm}$ e até $5 \mathrm{~cm}$ em sua maior dimensão \\
\hline T3 & Tumor com mais de $5 \mathrm{~cm}$ em sua maior dimensão \\
\hline \multirow[t]{5}{*}{ T4 } & Tumor de qualquer tamanho, com extensão direta à parede torácica ou à pele \\
\hline & T4a - extensão para parede torácica \\
\hline & $\begin{array}{l}\text { T4b - edema (incluindo peau d'orange) ou ulceração da pele da mama ou nódulos cutâ- } \\
\text { neos satélites, confinados à mesma mama }\end{array}$ \\
\hline & T4c - T4a e T4b associados \\
\hline & T4d - carcinoma inflamatório \\
\hline \multicolumn{2}{|c|}{ N - Linfonodais regionais } \\
\hline NX & Os linfonodos regionais não podem ser avaliados (por ex. foram removidos previamente) \\
\hline NO & Ausência de metástases nos linfonodos regionais \\
\hline N1 & Metástase em linfonodo(s) auxiliar(es) homolateral (is) móvel (is) \\
\hline N2 & Metástase nos linfonodos axilares homolaterais fixos uns aos outros ou a outras estruturas \\
\hline N3 & Metástase nos linfonodos da cadeia mamária interna homolateral \\
\hline \multicolumn{2}{|c|}{ M - Metástases a distância } \\
\hline $\mathrm{MX}$ & A presença de metástases a distância não pode ser avaliada \\
\hline MO & Ausência de metástases a distância \\
\hline M1 & Metástases a distância (incluindo as metástases nos linfonodos supraclaviculares) \\
\hline
\end{tabular}

Figura 01- Classificação Clínica do Câncer de Mamam pelo sistema TNM

Fonte: BARROS et al ${ }^{11}$ 
Estadiamento do câncer de mama em função das diversas combinações possíveis pelo sistema TNM

\begin{tabular}{|llll|}
\hline Estadio 0 & Tis & N0 & M0 \\
\hline Estadio I & T1 & N0 & M0 \\
\hline Estadio Ila & T0 & N1 & M0 \\
& T1 & N1 & M0 \\
\hline Estadio Ilb & T2 & N0 & M0 \\
& T2 & N1 & M0 \\
\hline Estadio IIla & T3 & N2 & M0 \\
& T0 & N2 & M0 \\
& T1 & N2 & M0 \\
\hline Estadio IIlb & T2 & N1, N2 & M0 \\
& T3 & qualquer N & M0 \\
\hline Estadio IV & T4 & N3 & M0 \\
\hline
\end{tabular}

Figura 02- Estadio do Câncer de Mama

Fonte: BARROS et al ${ }^{11}$

\section{Manifestações clínicas}

Na grande maioria dos casos, os sintomas do câncer de mama são descobertos pela paciente, por meio do autoexame. Dentre as manifestações clínicas comumente encontradas, enumera-se a presença de nódulo mamário, espessamento de pele, descarga papilar atípica, dor ${ }^{1}$.

O nódulo palpável, geralmente, é identificado pela paciente acometida pelo câncer. Fato este a faz ir em busca de auxílio de profissional de saúde para elucidação diagnóstica. Paralelamente, pode ocorrer um espessamento em área adjacente ao nódulo, o que constitui alteração de consistência, uma área mais endurecida ${ }^{12}$

A descarga papilar fora do período gravídico puerperal , por sua vez, deve ser analisada de maneira criteriosa, nas seguintes nuances: espontânea ou a expressão, uni ou bilateral, coloração alterada. Caso tenha as características de ser espontânea, unilateral, de ducto único, sanguinolenta há fortes indícios de malignidade 12 .

\section{Tratamento}

O tratamento do câncer de mama localizado está inferido, inicialmente, na cirurgia. Esta possui como finalidades proporcionar o controle locorregional, o prognóstico, a orientação de terapêutica e a máxima conservação do órgão ${ }^{13}$.

Em se tratando das cirurgias, pode-se dividi-las em cirurgias de todo o corpo glandular mamário e as conservadoras (retirada parcial do órgão). A cirurgia conservadora da mama para tumores em fase inicial, seguida de radioterapia, fornece resultados semelhantes quando comparado à cirurgia radical ${ }^{13,14}$.
Em estadios superiores a II, de forma individualizada, utilizar-se-á quimioterapia, radioterapia e hormonioterapia ajuvantes, responsáveis por diminuir o risco de recidiva. Em doença disseminada, a quimioterapia é o que se estabelece como tratamento oncológico ${ }^{14}$.

\section{Ambiente e câncer de mama}

A redução da incidência de câncer de mama é uma das prioridades inerentes à Saúde Pública, contudo, encontrar maneiras de concretizar esse fato é um desafio ímpar. Como em grande parte dos tipos de câncer no adulto, a neoplasia maligna de mama é moldada para se desenvolver a partir de danos cumulativos induzidos por desencadeadores internos e externos, resultando em eventos cancerígenos iniciais, com progressão das células e tecidos afetados, por meio dos múltiplos estágios integrativos (organismo e meio ambiente) ${ }^{15}$.

Atualmente, existe um vasto corpo de evidências sobre a relação entre o câncer de mama e a variedade de fatores externos que podem ser considerados como constituindo o ambiente. A informação a despeito das interações entre susceptibilidade genética e elementos ambientais gera uma maior aproximação das ciências naturais e aplicadas acerca das análises de estudos epidemiológicos, experimentais e mecanicistas da biologia do câncer de mama, direcionados a elucidação de como os fatores externos podem influenciar a etiologia da referida nosologia ${ }^{15}$. 


\section{Fatores de risco}

A literatura sobre os fatores de risco para câncer de mama, em particular, é grande e variada.

Dentre os fatores de risco para o desenrolar de câncer de mama, consideram-se como principais: idade avançada (maior do que 60 anos), nuliparidade, menarca precoce (até os 11 anos de idade), menopausa tardia (acima de 55 anos), primeira gestação após os 28 anos, uso de contraceptivos por período acima de cinco anos, terapia de reposição hormonal (TRH), história familiar e pessoal positivas, hábitos de vida e influências ambientais ${ }^{16}$

Em se tratando de história familiar e pessoal, infere-se: um ou mais parentes de primeiro grau com câncer de mama antes dos 50 anos, um ou mais parentes de primeiro grau com câncer de mama bilateral ou câncer ovariano em qualquer idade, parente com câncer de mama masculina, câncer de mama ou doença mamária benigna prévios ${ }^{16}$.

Ao se tratar de fatores externos, detém-se o estilo de vida e ambiente. Os hábitos de vida com maior probabilidade de fomentar o desenvolvimento do câncer ora em tela são a obesidade, uso regular de álcool acima de 60 gramas por dia, tabagismo. No fator ambiental, tem-se a exposição prévia às radiações ionizantes, diretamente proporcional à dosagem da radiação e inversamente proporcional à idade da mulher na época da exposição ${ }^{17}$.

\section{Impacto Socioeconômico do Câncer de Mama}

Em uma das vertentes do fator conceitual ambiente, encontra-se a repercussão econômica que pode ser gerada pelo câncer de mama.

\section{Declaração de conflitos de interesses}

Os autores do artigo afirmam que não houve nenhuma situação de conflito de interesse, tais como propostas de financiamento, emissão de pareceres, promoções ou participação em comitês consultivos ou diretivos, entre outras, que pudessem influenciar no desenvolvimento do trabalho.

\section{Referências}

1- COSTA, J.M.M. Qualidade de Vida na Mulher em Quimioterapia por Cancro da Mama: Estudo de Coorte Prospectivo. 2010. 160 f. Dissertação (Mestrado) - Curso de Mestrado em Oncologia, Instituto de Ciências Biomédicas Abel Salazar, Universidade do Porto, Porto, 2010. Disponível em: https://repositorioaberto.up.pt/bitstream/10216/26920/2/QdV em mulheres com ca da mama em qt Estudo de coorte prospectivo.pdf. Acesso em: 23/10/2016.
O gasto atual com saúde no Brasil é de, aproximadamente, 9\% do PIB per capita . Dados da Organização Mundial de Saúde apontam gastos de 990 dólares per capita no ano de 2010, no Brasil, destes $47 \%$ são financiados pelo governo ${ }^{18}$.

SIQUEIRA et al ${ }^{18}$ evidenciaram que os gastos diretos totais ocorridos por neoplasia de mama foram do valor de R\$ 302 milhões, no ano de 2008, para R\$ 633 milhões, no ano de 2015. Os gastos com quimioterapia representam $68 \%$ do total gasto, as internações hospitalares, $18 \%$; as cirurgias oncológicas, $10 \%$; e os benefícios previdenciários, $4 \%$.

Infere-se que quanto maior o investimento para a detecção precoce da referida neoplasia, maior será a sobrevida e a qualidade de vida das mulheres, o que originará menor impacto econômico e social para o país.

\section{Conclusão}

O Câncer de mama detém altas taxas de mortalidade, devido à heterogeneidade da doença e a exposição diferenciada dos pacientes a diversos fatores ambientais, mesmo com a tecnologia e evolução dos tratamentos quimioterápicos. Em sua maioria, os diagnósticos são confirmados em estadio avançado, em mulheres ativas economicamente, com nível intermediário de escolaridade. Mesmo diante de tal cenário, essa neoplasia pode ser prevenida evitando os fatores de risco, que, geralmente, estão ligados a ordem socioambiental. Urge necessidade de maiores estudos e investimentos acerca de tal problemática, por se constituir, na atualidade, de um imbróglio à saúde pública.

2- SCLIAR, M. História do conceito de saúde. Physis, Rio de Janeiro , v. 17, n. 1, p. 29-41, 2007 .Disponível em http://www.scielo.br/scielo.php?script=sci_arttext\&pid=S0 103-73312007000100003\&lng=en\&nrm=iso. Acesso em 23/10/2016.

3- WATSON, J.D;CRICK, FH "Molecular structure of nucleic acids: a structure for deoxyribose nucleic acid." Am J Psychiatry. New York, 160(4), 623-624,2003

4- ALEGRANCE, F.C. Qualidade de vida e estratégias de enfrentamento de mulheres com linfedema após câncer de mama. 2006. Dissertação (Mestrado em Ciências Médicas). Universidade Metodista da São Paulo, São Paulo. 2006

5- NOBRE, R.M.B et al. Perfil clínico-epidemiológico das neoplasias ocorridas no período de 2005-2012 no Estado do Paraná. Revista de Epidemiologia e Controle de Infecção, Santa Cruz do Sul, 2016. Disponível em: https://online.unisc.br/seer/index.php/epidemiologia/article/ view/8189/5189. Acesso em: 23/10/2016. 
6- GARCIA, S.N. Qualidade de vida de mulheres com neoplasia mamária em tratamento quimioterápico.2014. 104 f. Dissertação (Mestrado) - Curso de Mestrado em Enfermagem, Departamento de Enfermagem, Universidade Federal do Paraná, Curitiba, 2014. Disponível em: http://acervodigital.ufpr.br/bitstream/handle/1884/37387/R

$$
\text { - } \quad \text { D } \quad-\quad \text { SABRINA } \quad \text { NUNES }
$$

GARCIA.pdf?sequence=1\&isAllowed=y. Acesso em: 23/10/ 2016.

7- PAIVA, S.M.M. Avaliação da Qualidade de Vida de Pacientes Oncológicos em Tratamento Quimioterápico Adjuvante. 2006. 111f. Dissertação ( Mestrado em Enfermagem). Escola de Enfermagem de Ribeirão Preto, Universidade de São Paulo, Ribeirão Preto. 2006

8- NICOLUSSI A.C; SAWADA N.O. Qualidade de vida de pacientes com câncer de mama em terapia adjuvante. Rev Gaúcha Enferm., Porto Alegre. v 32(4),p 759-66,2011.

9- INSTITUTO NACIONAL DE CÂNCER JOSÉ ALENCAR GOMES DA SILVA. Coordenação de Prevenção e Vigilância Estimativa 2016: incidência de câncer no Brasil / Instituto Nacional de Câncer José Alencar Gomes da Silva - Rio de Janeiro: INCA, 2015

10- SILVA, C.R.M; LELIS, M.C; MONTEIRO, S.O. Estadiamento do Câncer de Mama. In: FIGUEIREDO, Euridice; MONTEIRO, Mauro; FERREIRA, Alexandre. Tratado de Oncologia. Rio de Janeiro: Revinter, 2013. Cap. 103. p. 1009-1013.

11- BARRos A, BARBOSA E, GEBRIM L. Projeto Diretrizes: Diagnóstico e Tratamento do Câncer de Mama. 1 ed. Brasília: Conselho Federal de Medicina; 2001.
12-CANELLA, E.O. Métodos diagnósticos por Imagem: Mamografia. In: FIGUEIREDO, E; MONTEIRO, M; FERREIRA, A. Tratado de Oncologia. Rio de Janeiro: Revinter, 2013. Cap. 106. p. 1025-1034.

13- DE SOUSA, Maianna Macêdo; FIGUEREDO, Sabrina Barreto; FERNANDES, Reinaldo Magalhães. Perfil clínicoepidemiológico de mulheres com neoplasia de mama atendidas no Hospital Regional de Referência no município de Araguaína-TO no período de 2000 a 2015. DESAFIOS, v. 2 , n. 2 , p. $283-306,2016$

14- BARROS A, KATZ A, JARDIM D, BASTOS D. Tratamento de Tumor de Mama Localizado. In: HOFF P, KATZ A, CHAMMAS R, FILHO V, NOVIS Y. Tratado de Oncologia. São Paulo: Atheneu Editora; 2013. p. 20592065.

15- IOM ( Institute of Medicine). Breast Cancer and the environment: A life cause approach. Washington, DC: The National Academy Press, 2012

16- SILVA, P.A; RIUL, S. Câncer de mama: fatores de risco e detecção precoce. Revista Brasileira de Enfermagem, Brasilia, v. 6, n. 64, p.1016-1021, 2011. Disponível

http://www.scielo.br/pdf/reben/v64n6/v64n6a05.pdf. Acesso em: 23/10/ 2016.

17- MINISTÉRIO DA SAÚDE (BR). Instituto Nacional do Câncer. Ações de enfermagem para o controle do câncer. 3 ed. Rio de Janeiro: INCA; 2008.

18. SIQUEIRA, Alessandra de Sá Earp et al. Impacto econômico das internações, quimioterapias e afastamentos por Neoplasia Maligna de Mama no Brasil. DIVERSITATES International Journal, v. 8, n. 1, 2016. 